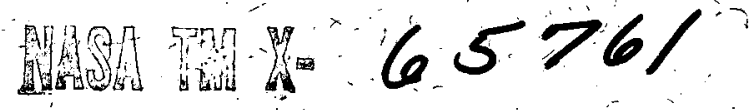

\section{LONG TERM TEMPORAL VARIATIONS OF THE HARD X-RAY FLUX FROM THE CENTAURUS REGION}

\section{DANIEL A. SCHWARTZ LAURENCE. E. PETERSON HUGH S. HUDSON}
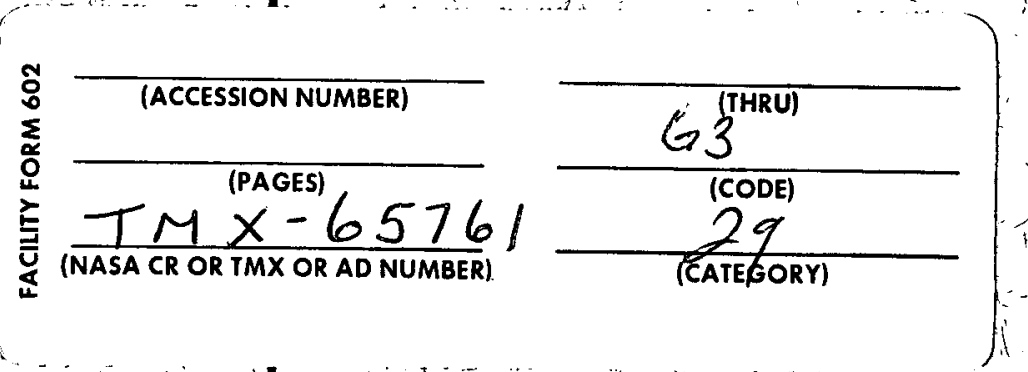

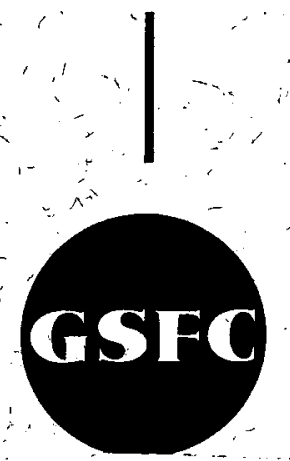

N72-12802

Unclas 09312
Reproduced by

NATIONAL TECHNICAL

INFORMATION SERVICE

U S Department of Commerce
Springfield VA 22151

\section{NOVEMBER 1971}

\section{GODDARD SPACE FLIGHT CENTER \\ GREENBELT, MARYLAND}

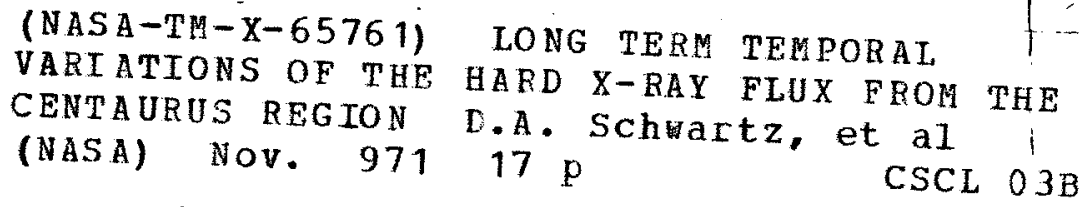


LONG TERM TEMPORAL VARIATIONS OF THE HARD

$X$-RAY FLUX FROM THE CENTAURUS REGION

\author{
Danie1 A. Schwartz* \\ Goddard Space Flight Center, Greenbelt, Maryland 20771 \\ Laurence E. Peterson \& Hugh S. Hudson \\ University of California at San Diego, La Jo1la, Calif. 92037
}

\title{
ABSTRACT
}

The University of California at San Diego (UCSD) X-ray telescope aboard the third Orbiting Solar Observatory (OSO-III) observed the Centaurus region daily from 1967 October to 1968 February, and also for five days in 1968 June. For this period, we derive a stable minimum flux of $0.33 \pm 0.03$ photons $\left(\mathrm{cm}^{2} \mathrm{sec}\right)^{-1}$ between 7.7 and $38 \mathrm{keV}$ from a source around $l=305^{\circ}$. Several single days show enhanced fluxes, and two extensive flaring episodes, one with a soft and the other a very hard spectrum, last at least ten days.

\footnotetext{
*NAS-NRC Resident Research Associate
} 
I. INTRODUCTION

The OSO-III X-ray telescope observed the Centaurus region almost daily for four months between October 1967 and February 1968, and during five days in June 1968. Assuming one single source at $\ell=305^{\circ}, \mathrm{b}=1.5^{\circ}$, as measured from balloons by Lewin, Clark, and Smith (1968b) on 1967 October 15 and 24, our counting rates during October 1967 are consistent with the average strength and spectrum (extrapolated to $10 \mathrm{keV}$ ) of those balloon measurements. The single source at this position also explains much of our counting rate over the entire period of observation, and we find this source to have a generally constant flux when averaged over one day. We also see two periods of flaring on at least a 10 day time scale, and several single days of enhanced fluxes. We cannot directly determine the origin of these flares. They quite possibly arise from any of several known variable sources (Lewin, et al. 1971; McClintock et al. 1971; Cooke \& Pounds 1971; Giacconi et a1. 1971; Margon et a1. 1971) within $15^{\circ}$ of the above position. We have spectral measurements of both the steady and flaring emission spanning the energy range observable from rockets to that observable from balloons.

The source observed by the MIT balloon survey (Lewin, Clark and Smith 1968a) was originally identified with Cen XR-2, a source which flared up in Spring 1967 by a factor of $>50$ in the 2-8 keV range and then decayed with about a 25-day e-folding time. The several original rocket results gave the position of this source as $\ell=307^{\circ}$ to $310^{\circ}$, with typical error circles of $3^{\circ}$ (Chodil et al. 1967; Francey et a1. 1967; Cooke et al. 1967). A recent reanalysis of one of the April 1967 
$-3-$

rocket flights gives a galactic longitude $310.2 \pm 1.0^{\circ}$ (Francey 1971), incompatible with the balloon position. We will use the revised designation GX 304-1 (McClintock et a1. 1971) for the basic source which we observe.

\section{EXPERIMENTAL DETAILS}

The UCSD X-ray telescope (Hicks, Reid, and Peterson 1965) aboard OSO-III consists of a $9.6 \mathrm{~cm}^{2} \mathrm{NaI}(\mathrm{T} 1)$ scintillator actively collimated by a concentric CsI(T1) cylindrical she 11 to a $50 \%$ response radius of $11.5^{\circ}$. The satellite telemeters 5 useful channels of pulse height analysis, logarithmically spaced between 7.7 and $113 \mathrm{keV}$. The basic data consist of arrival directions of individual photons in each energy channel; these are reduced to true count rates averaged over bins $6^{\circ}$ along the scan circle, as described by Schwartz, Hudson, and Peterson (1970). The one difference is that the data here are averaged over only 1 day, which represents a negligible precession of only $1^{\circ}$ on the sky perpendicular to the scan circle. From the aspect solution and the satellite spin rate, we calculate the total effective response in $\left(\mathrm{cm}^{2} \mathrm{sec}\right)$ for each bin along the scan circle, to any given position of a cosmic X-ray source. Because of our large aperture and the uncertainty arising from digitizing the arrival direction, we make no attempt to derive positions of $\mathrm{X}$-ray sources from our data alone.

An error of $3^{\circ}$ in the adopted source position would change our derived fluxes by 1 to $20 \%$ during certain periods of observation, but would not change our general conclusions. In particular, the observed flaring episodes cannot be attributed to aspect errors or sudden changes in the satellite spin axis. We use only data which contain less than about $10 \%$ 
contamination from other nearby known sources, based on previous surveys of this region (Lewin, Clark, and Smith 1968a; Cooke and Pounds 1971) and assuming the other sources do not vary. The breakdown of this assumption may explain the observed flares. The existence of a constant minimum flux, reported below, indicates that most of our counts do come from the vicinity of GX 304-1.

From the raw true count rate we separately subtract corrections for background induced by radioactivity and by cosmic rays, and then subtract a correction due to the previously determined diffuse component of cosmic $\mathrm{X}$-rays. The more straightforward procedure of estimating background from the same scan with the telescope pointing off the source is not applicable because different parts of this circle receive sky exposure during different parts of the orbit, and because when several sources are simultaneously on the scan circle we do not accumulate sufficient off-source background statistics. The danger of contamination of the counting rates by geomagnetically trapped particles made it necessary to reject a large fraction of the raw data. By studying the background over the 16 months of satellite operation, we estimate that these corrections are known to better than $10 \%$ (Schwartz 1969). The background rate summed over the 3 lowest channels equals about 7.7 counts per second, of which $6.5 \mathrm{sec}^{-1}$ arise from the diffuse cosmic $x$-rays. We measure additional rates of 3 to 10 counts per second from Centaurus. Because of this large signal to background ratio the present data have about the same statistical significance that previous observers have obtained at the same energies, even though our telemetry limits us to about 100 to 200 (cm sec) $^{-1}$ effective daily exposure per energy channel. 


\section{OBSERVATIONS}

Figure 1 presents data from our coverage of Centaurus. The bottom points represent the one-day averages of the integral flux from GX 304-1, with background subtracted, from our three lowest channels in units of counts $\left(\mathrm{cm}^{2} \mathrm{sec}\right)^{-1}$ between 7.7 and $38 \mathrm{keV}$. The error bars shown are based solely on counting statistics and are not connected for a few points of less than $2 \sigma$ significance. We also show the integrated fractional. exposure per spin revolution to GX 304-1, which we calculated by taking account of the declination of the source from the scan circle. The error bars show the change in exposure resulting from $a \pm 2^{\circ}$ error in the aspect solution. Such an aspect error would represent about a $2 \sigma$ deviation from the average solutions determined independently by the MIT group (Clark 1968) and at UCSD. A given percentage error in the true exposure would produce the same percentage error in the integral flux; note that such errors are not symmetric about the mean.

The oso scan circle on the celestial sphere is inclined by about $60^{\circ}$ to the equator, and automatically precesses about $1^{\circ}$ per day in right ascension to follow the apparent solar motion. It is the chance declination of GX $304-1$ at about $-60^{\circ}$ which allows it to stay within our scan so long (see Fig. 9 of Peterson 1970). For comparison, we have data on the Crab nebula for 20 days between 1967 December 3, and December 29. It appears to have a constant flux of 1.41 photons $\left(\mathrm{cm}^{2} \mathrm{sec}\right)^{-1}$ between 7.7 and $38 \mathrm{keV}$. The root-mean-square deviation for a single day is $8.5 \%$ and the maximum deviation of $18 \%$ occurs on the day when the Crab is calculated to be the furthest from the scan circle, and thus most susceptible to aspect errors. 
Figure 1 shows two definite, extended episodes of flaring; 1967 October 31 to November 14 and 1968 January 23 to February 2. Individual days, such as 1967 November 19, 21 and 23, December 30, and 1968 Jan. 4 and 24 show fluxes of 2.5 to $5 \sigma$ above surrounding days. For such enhancements, we do not have other evidence, such as the systematic decline of counting rate for the extended flares, to rule out possible charged particle contamination of the X-ray telescope. Actually, our daily average does not represent a uniform sample throughout the day, but rather contains all the data during each day that happened to occur at times consistent with background selection criteria. Thus these single day enhancements could have occurred on the same time scales, ten minutes to a few hours, as flares observed from Sco X-1 (Hudson, Peterson, and Schwartz 1970; Lewin et al. 1970) and from a source at $\ell \sim 301^{\circ}$. (Lewin et a1. 1971; McClintock et a1. 1971).

Ignoring $3 \sigma$ deficits on October 25 and 30 and a $3 \sigma$ increase on February 11, the flux during each interval denoted by a bar at the bottom of Figure 1 is statistically consistent with an assumed constant source. Also, the average flux over each of these intervals is barely consistent (to $4 \%$ confidence by a $x^{2}$ test) with a constant received "base-line" flux from GX 304-1 of $0.33 \pm 0.03$ photons $\left(\mathrm{cm}^{2} \mathrm{sec}\right)^{-1}$ (corrected for detector response) between 7.7 and $38 \mathrm{keV}$. Satellite observations allow analysis for periodic variations of the order of a few days, such as might arise from eclipsing binaries. During the constant intervals we derive an upper limit of $5 \%$ for a fraction of the emission associated with a sine wave with a period between 4 and 20 days. 
The statistics during one day do not suffice to give spectral information, so we average over each of the intervals shown in Figure 1. The resulting data appear in Table 1 . Figure 2 shows spectra1 measurements from this region. OSO-III "quiet" data are the average of lines $1,6,8,11$ and 12 in the Table. We also show the lower energy measurements from Cen XR-2, which have generally been interpreted as thermal bremstrahlung radiation because of the exponential energy spectrum observed in the original source flareup (Chodil, et al. 1967). The present data fit such spectra very we11, although power law spectra of number index $2.2 \pm 0.3$ could fit the data as well from at least 7.7 to $40 \mathrm{keV}$. In our highest energy channel we sometimes see an excess such as that reported by Lewin et al. (1968a). This excess varies independently of the 7 to $40 \mathrm{keV}$ flux, decreasing between October 1957 and January 1968 and then increasing to a maximum in February 1968. Note the sharp cutoff in channel five of the "hard flare" spectrum (Table 1, line 9).

IV. DISCUSSION

Figure 3 shows $\mathrm{X}$-ray sources in the Centaurus region, along with the OSO-III scan circles at different times. The source symbols generally represent a $1 \sigma$ position uncertainty, except Cen $A$ and Cen X-3 have been measured by UHURU to greater precision than shown (Kellogg et a1. 1971; Giacconi et al. 1971). Although our 50\% response circle is quite large, for a constant source to give a steady derived flux, to better than $30 \%$ as we scan the region over many different paths, it must locate within 
about $4^{\circ}$ of our assumed position of GX 304-1. The sources GX 301-2 and Cen XR-2 would be candidates for the source of the steady emission, except for their absence in 1967 October (Lewin, Clark and Smith 1968a). The flares might be due to Cen X-3, Cen X-5, GX 301-2, or Cen XR-2, a11 of which are known to be highly time variable. The exact flare magnitude would then depend on the relative exposure factor of GX $304-1$ and the actual flare-source position.

The November flare has a much lower temperature than the steady emission, and thus is presumably not connected with it. Subtracting the steady component gives an effective temperature of about 7 or $8 \mathrm{keV}$. This flare spectrum, extrapolated below $10 \mathrm{keV}$, gives a flux somewhat in excess of the measurements attributed to Cen XR-2 by Rao et a1. (1969) on 1968 November 3 and November 7. MacGregor et a1. (1970) report that Cen XR-2 was not detectable just 15 hours after the first flight of Rao et al. We see no inconsistency between these two observations in view of the rapid variations we find, for example, in the interval 1967 November 16 and 24. Note that these authors were actually assuming a source position of $\ell=305^{\circ}$ to $307^{\circ}$.

The e-folding decay times for the two flares we observe are on the order of ten days, probably consistent with the 25 day decay time of the lower energy $X$-rays in Spring of 1967. The rise times may also a11 be about 1 day. (Although the 2-8 keV outburst was not directly observed for Cen XR-2, Conner, Evans, and Belian (1969) found a rise time of a few days for the similar burst from Cen XR-4). In spite of the rough similarities of their time profiles, the various outbursts from this region had appreciably different amplitudes and spectral behavior! 


\section{ACKNOWLEDGEMENTS}

We thank again the many individuals at Ball Brothers Research Corp., Goddard Space Flight Center, and the University of California at San Diego for construction of the satellite and the $X$-ray telescope, management of the OSO project, and assistance in the data analysis. Work at UCSD was supported by NASA contracts NAS 5-3177 and NSG-318. 


\section{FIGURE CAPTIONS}

FIGURE 1: OSO-III observations of Centaurus. Top points: calculated fractional exposure to GX304-1, with error bars corresponding to a $2^{\circ}$ aspect error. Bottom points: total flux summed over 7.7 to $38 \mathrm{keV}$ range and averaged over each day. Bottom bars: periods of time-averaging to obtain spectral information.

FIGURE 2: Spectral data from Centaurus. OSO-III "quiet" refers to the intervals indicated in Figure 1. The "soft" and "hard" flares represent the November and January flareups, respectively. MIT 1967 data are averaged over their 15 and 24 October balloon flights.

FIGURE 3: OSO-III scans of the Centaurus region, together with known $\mathrm{X}$-ray sources. The ellipses and diamonds are variable sources observed by the MIT balloon group. We identify the "steady" source of OSO-III observations with the MIT source around $\ell=304^{\circ}$. Cen $X-3$, Cen $X-5$, Cen XR-2, Cen XR-4, and Lup XR-1 are a1so known to be variable sources. 


\section{REFERENCES}

Chodi1, G., Mark, H., Rodrigues, R., Seward, F., Swift, C.D., Hiltner, W.A., Wallerstein, G., and Mannery, E.J. 1967, Phys. Rev。Letters, 19,681 .

Chodil, G., Mark, H., Rodrigues, R., and Swift, C.D. 1968, Ap. J。 (Letters), 152, I45.

Clark, G. 1968 (private communication).

Conner, J.P., Evans, W.D.; and Belian, R.D. 1969, Ap. J. (Letters), 157, L157.

Cooke, B.A., and Pounds, K.A. 1971, Nature, Phys. Sci., 229, 144.

Cooke, B.A., Pounds, K.A., Stewardson, E. A, and Adams, D.J. 1967, Ap。 J. (Letters), 150, L189.

Francey, R.J. 1971, Nature Phys. Sci., 229, 229.

Francey, R.J., Fenton, A.G., Harries, J.R., and McCracken, K.G., 1967, Nature, 216, 773 .

Giacconi, R., Gursky, H., Kellogg, E., Schreier, E., and Tananbaum, H. 1971, Ap. J。 (Letters), 167, I67.

Hicks, D.B., Reid, L., Jr., and Peterson, L.E. 1965, IEEE Trans. Nuc1. Sci., NS-12, 54 .

Hudson, H.S., Peterson, L.E., and Schwartz, D.A. 1969, Ap. J. 157, 389.

Hudson, H.S., Peterson, L.E., and Schwartz, D.A. 1970, Ap. J. (Letters), 159, L51.

Ke1logg, E., Gursky, H., Leong; C., Schreier, E., Tananbaum, H., and Giacconi, R。1971, Ap. J. (Letters), 165, I49.

Lewin, W.H.G., Clark, G.W., and Smith, W.B. 1968a, Ap. J. (Letters), 152, 149 .

$1968 \mathrm{~b}$, Nature, 219, 1235.

Lewin,, W.H.G., McClintock, J.E., Ryckman, S.G., Glass, I.S., and Smith, W.B. 1970, Ap. J. (Letters), 162, L109.

Lewin, W.H.G., McClintock, J.E., Ryckman, S.G., and Smith, W.B. 1971, Ap. J. (Letters), 166, L69. 
MacGregor, A., Seward, F., and Turie1, I. 1970, Ap. J., 161, 979.

Margon, B., Lampton, M., Bowyer, S., and Cruddace, R. 1971, Ap. J. (Letters), 169, L23.

McClintock, J. E., Ricker, G. R., and Lewin, W. H. G. 1971, Ap. J. (Letters), 166, L73.

Peterson, L. E. 1970, in Non-Solar X- and Gamma-Ray Astronomy, I.A.U. Symp. No. 37, ed. L. Gratton (Dordrecht-Holland: D. Reidel Publishing Co.), p. 59 .

Rao, U. R., Chitnis, E. V., Prakasarao, A. S., and Jayanthi, U. B. 1969, Ap. J. (Letters), 157, L127.

Schwartz, D. A. 1969, unpublished Ph.D. Thesis, Univ. of California at San Diego.

Schwartz, D. A., Hudson, H. S., and Peterson, L. E. 1970, Ap. J., 162, 431. 
TABLE 1

HARD X-RAY FLUXES FROM CENTAURUS

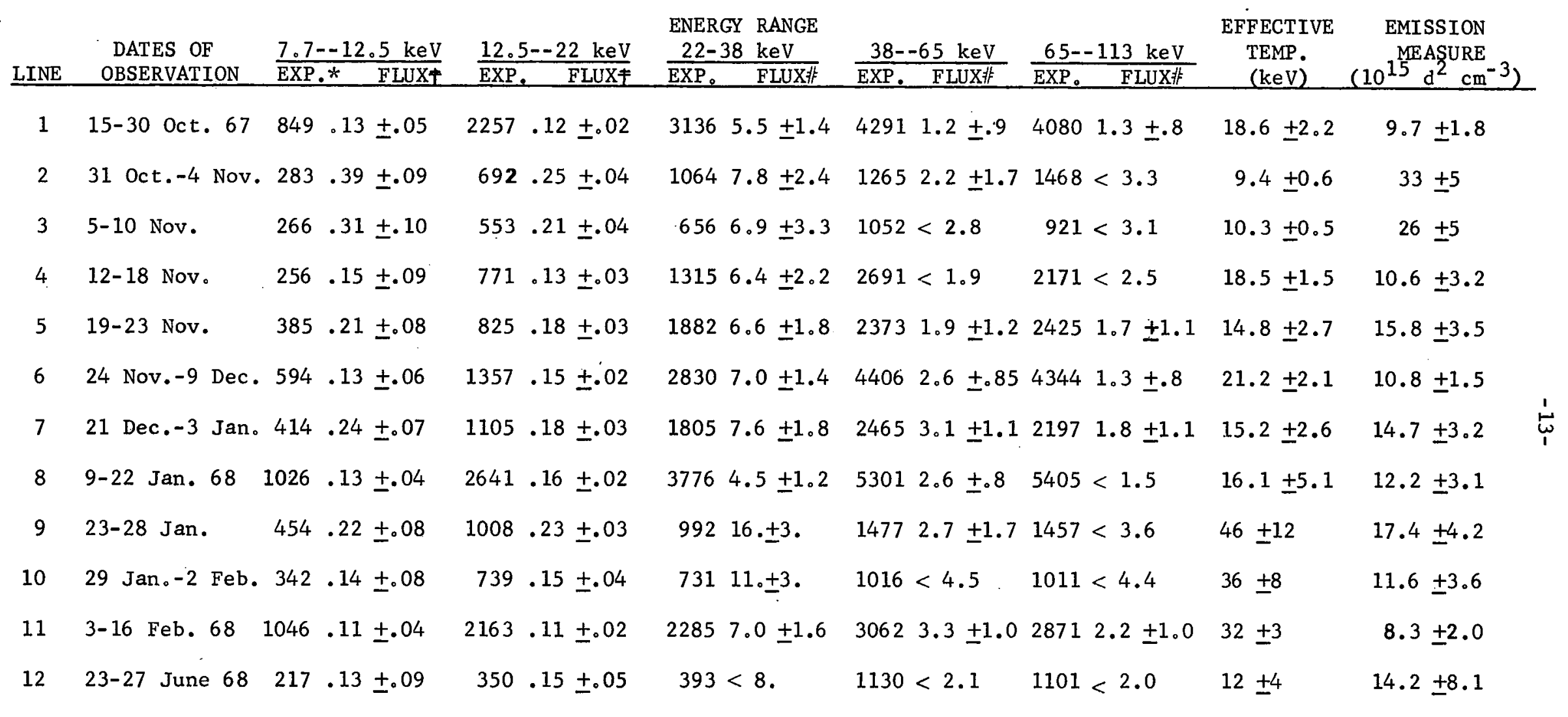

$*$ Exposure in $\left(\mathrm{cm}^{2}--\mathrm{sec}\right)$

* Flux in counts $\left(\mathrm{cm}^{2}--\mathrm{seq}\right)^{-1}$. Fluxes shown with error bars are more than $2 \sigma$ above background.

非 1 ux in $10^{-2}$ counts $\left(\mathrm{cm}^{2}--\mathrm{sec}\right)^{-1}$ 


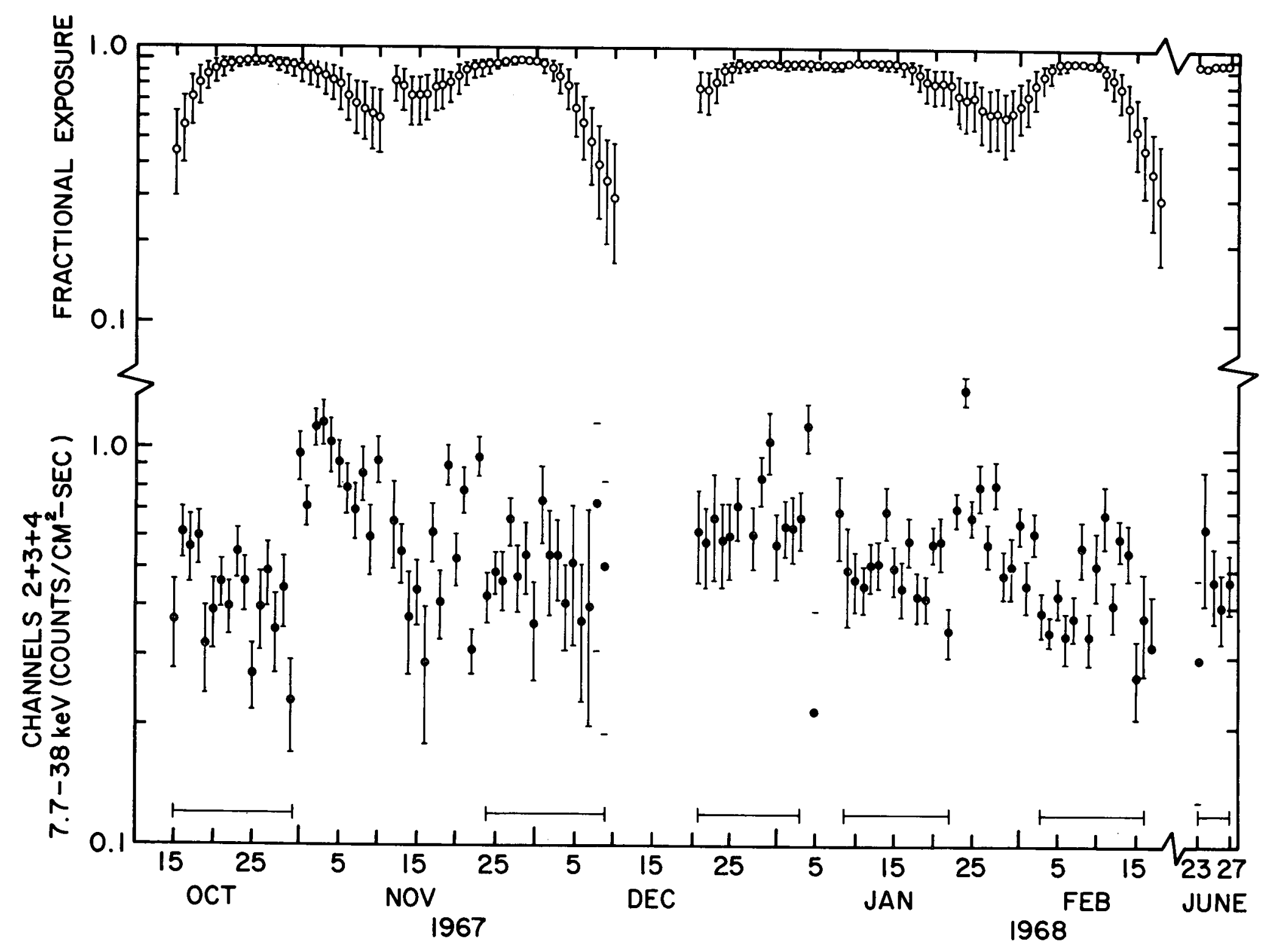




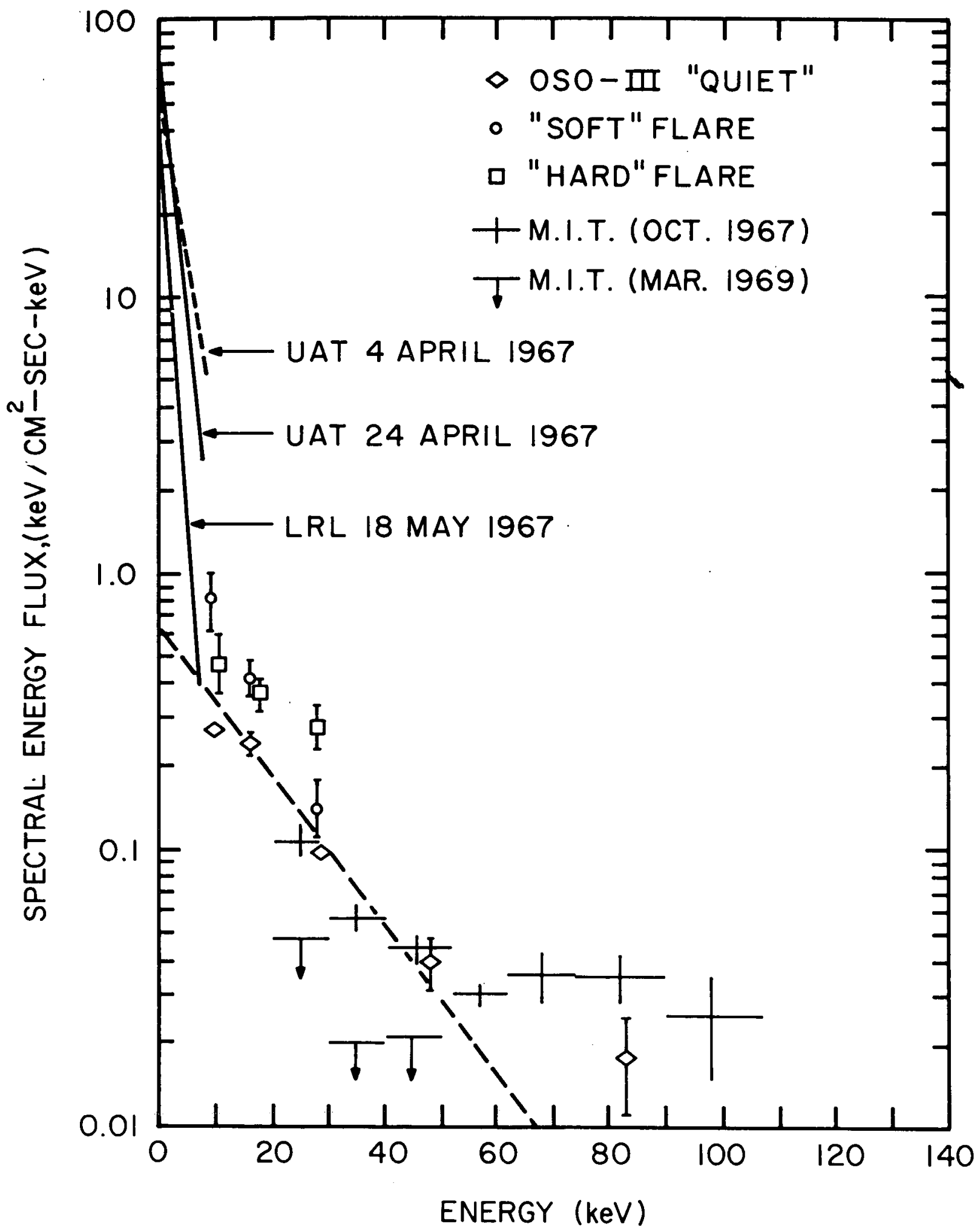




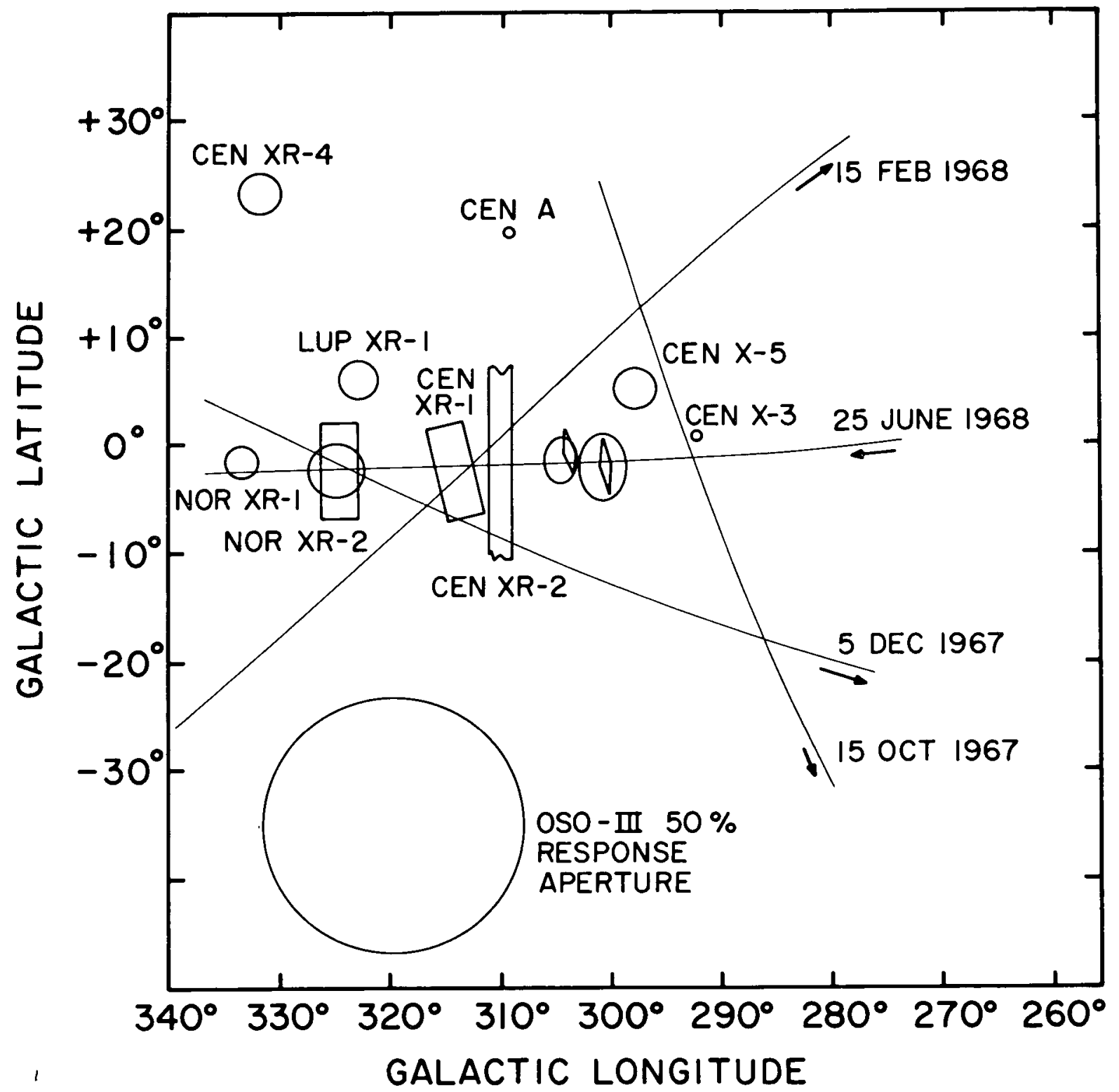

